\title{
PM NOISE GENERATED BY NOISY COMPONENTS
}

\author{
H. D. Ascarrunz, SpectraDynamics, Lafayette CO \\ Aimin Zhang, National Institute of Metrology, Beijing, China \\ E. S. Ferre-Pikal, NIST, Boulder CO \\ F. L. Walls, NIST, Boulder CO
}

\begin{abstract}
This paper investigates the phase modulation (PM) noise generated by intrinsic reactance fluctuations in signal carrying components. We report the fractional fluctuation in the reactance of commonly available capacitors, inductors and varactors. Fractional fluctuations were inferred by measuring the phase shift of the carrier signal across the components and the resulting PM noise due to intrinsic fluctuations. In addition, various methods to reduce the effect of intrinsic noise in low noise circuit applications are discussed.
\end{abstract}

\section{Introduction}

For a simple LCR circuit, the phase shift is,

$$
\phi=\arctan \left(\frac{i m Z}{r e Z}\right) \text {, }
$$

where $\mathrm{im} Z$ and re $Z$ are the imaginary and real parts of the impedance $Z$.

From (1),

$$
\Delta \phi=\frac{1}{1+\left(\frac{i m Z}{r e Z}\right)^{2}} \cdot \frac{\Delta i m Z(f)}{r e Z},
$$

where $\operatorname{\Delta im} Z^{2}(f)$ is the mean squared fluctuation in the reactance $Z$ at frequency $f$.

The power spectral density of phase modulation (PM) noise is defined as [1]

$$
S_{\phi}(f)=\frac{(\Delta \phi(f))^{2}}{B W},
$$

and relates to the single side-band PM noise by, [2]

$$
L(f) \cong \frac{1}{2} S_{\phi}(f) \text {. }
$$

From (1) \& (2),

$$
S_{\phi}(f)=\frac{\sin ^{2}(2 \phi)}{4} \cdot \frac{(\Delta i m Z(f))^{2}}{i m Z^{2}} \cdot \frac{1}{B W},
$$

The effect of reactance fluctuations on the phase noise scales with phase as $\sin ^{2}(2 \phi) / 4$. Therefore, by decreasing the angle across a reactive component it is possible to minimize the effect of its intrinsic reactive fluctuations on the $P M$ noise.
For $\phi$ small the PM noise can be approximated by

$$
S_{\phi}(f) \cong \phi^{2} \cdot \frac{(\Delta i m Z(f))^{2}}{i m Z^{2}} \cdot \frac{1}{B W} .
$$

In a circuit at resonance, (2) becomes

$$
\Delta \phi=\frac{\Delta i m Z(f)}{r e Z}
$$

since the $\mathrm{L}$ and $\mathrm{C}$ components of the imaginary impedance cancel and $\mathrm{imZ} \rightarrow 0$.

Thus, near resonance, from equations (3), (7), and given

$$
Q=\frac{1}{\omega C R}=\frac{\omega L}{R},
$$

a better approximation for the PM noise of a near resonant LCR circuit is

$$
S_{\phi}(f) \approx Q^{2} \cdot\left[\frac{(\Delta C(f))^{2}}{C^{2}}+\frac{(\Delta L(f))^{2}}{L^{2}}\right] \cdot \frac{1}{B W} ;(9)
$$

where $\Delta C^{2}(f)$ and $\Delta L^{2}(f)$ are the mean squared fluctuations in $C$ and $L$ at frequency $f$ and assuming that the fluctuations are independent.

\section{Phase Noise Measurement System}

A two channel cross-correlation system was used to measure the PM noise of our device under test (DUT) [Fig.1]. [1]

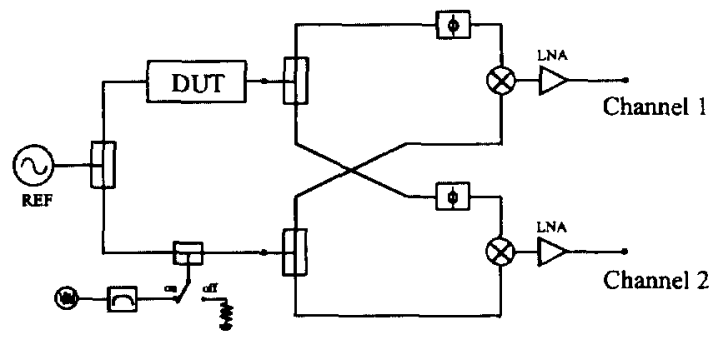

Figure 1: Two Channel Cross-Correlation Measurement System.

The low noise oscillator signal is split in two. One signal goes through the DUT. The other is summed with a noise source then beat with the signal out of the DUT in a phase detector. [2] 
Two parallel measurement channels are analyzed using cross correlation thereby decreasing the effects of the measurement system noise as $1 / \sqrt{N}$, where $\mathrm{N}$ is the number of measurements.

The noise source is used to calibrate the sensitivity of the mixers. [2]

\section{Results}

The effect of a component's intrinsic reactive fluctuations on the measured phase noise is related to the phase shift across the component by $\sin (2 \phi)$, as in (5). To maximize our measurement sensitivity to intrinsic reactance fluctuations, all our components were measured at a phase shift close to $45^{\circ}$. However, in low noise applications a small angle is desired. We took some of our measured
PM noise data and using equation (5), calculated the $5 \mathrm{MHz}$ PM noise for two angles; $45^{\circ}$ and $2.9^{\circ}$

[Fig. 2]. The PM noise induced by a component's intrinsic noise is $20 \mathrm{~dB}$ lower at $2.9^{\circ}$ (right axis) than at $45^{\circ}$ (left axis).

Near resonance, the PM noise scales as $Q^{2}(9)$. We measured and compared the $5 \mathrm{MHz} \mathrm{PM}$ noise of two LC filters. Figure 3 compares the effect intrinsic reactance fluctuations have on the PM noise of a circuit near resonance (pass-band) and a circuit far from resonance (notch). The capacitor data (PSD $\left.(\Delta C / C)^{2}\right)$ trace was included as a reference and has units of $\mathrm{dB} / \mathrm{Hz}$. The resulting PM noise of the passband filter was substantially higher than that of the notch.

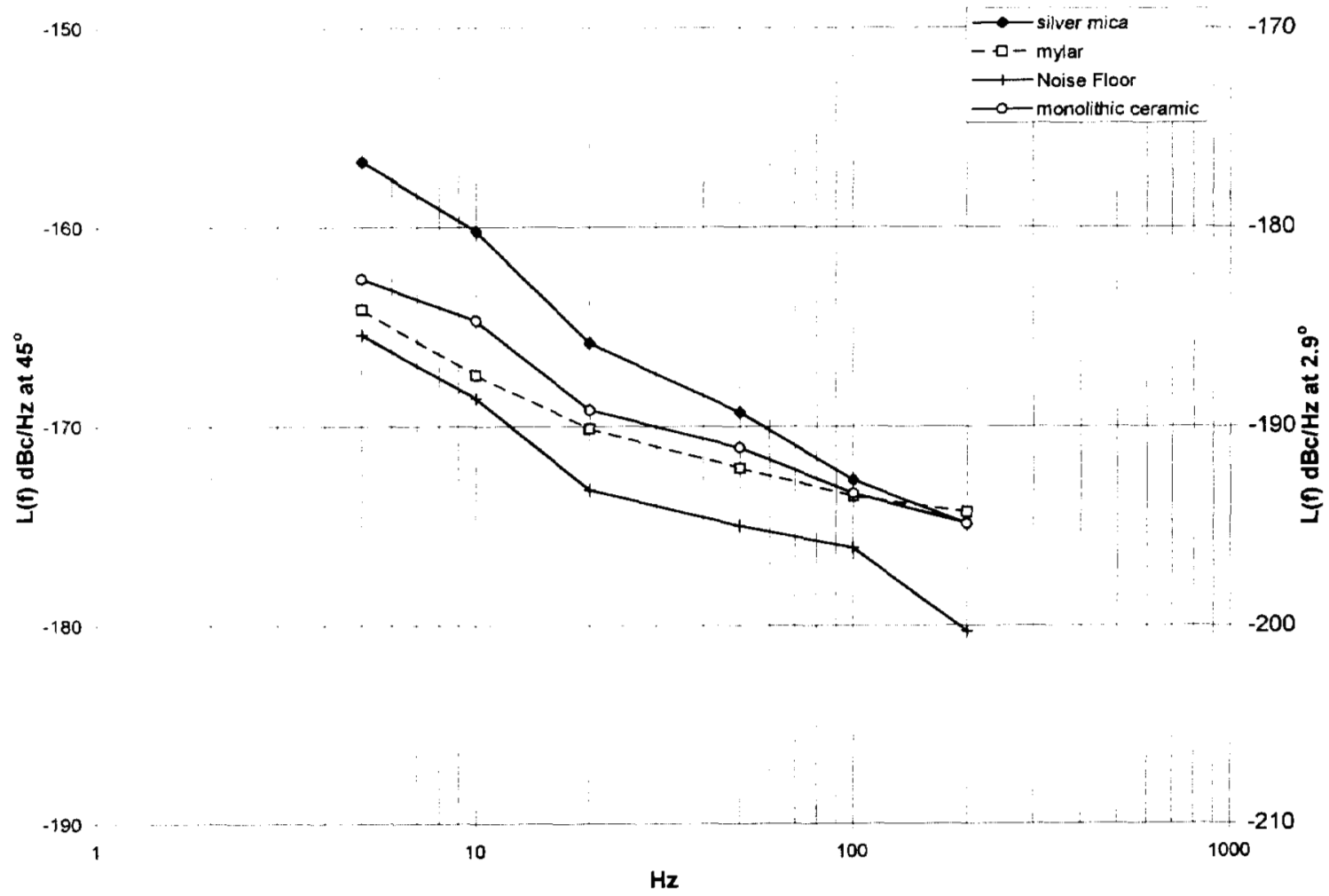

Figure 2: Projected PM noise at angles of $45^{\circ}$ and $2.9^{\circ}$; left and right axes respectively. 


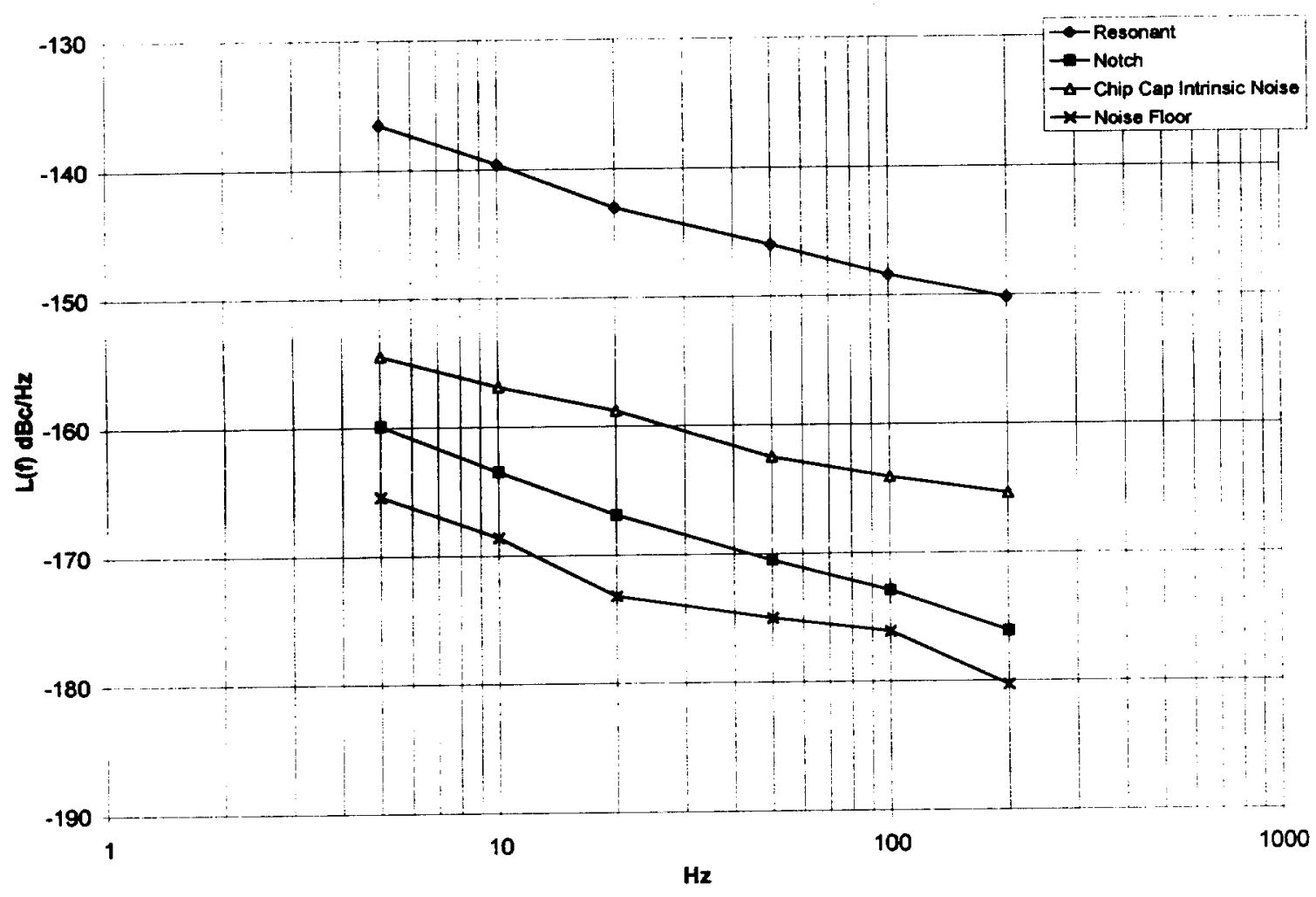

Figure 3: The $5 \mathrm{MHz}$ PM noise of two filter circuits, notch (far from resonance) and passband (near resonance).

The remainder of our paper deals with intrinsic reactive fluctuations in capacitors, inductors and varactors. To calculate the fractional fluctuations, the phase shift component was divided out, making it possible to provide an accurate comparison of the intrinsic reactive fluctuation of different commercial components. Components with different material composition were chosen to establish some guidelines when choosing components for low noise circuit design. Capacitors

Various families of capacitors were tested. Table 1 shows the different material and $5 \mathrm{MHz}$ phase information.

Figure 4 is a graph of $P S D(\triangle C / C)^{2}$ showing the intrinsic reactance fluctuations of different materials.

\begin{tabular}{|c|c|l|}
\hline Value (nF) & $\begin{array}{c}5 \mathrm{MHz} \\
\text { Phase shift } \\
\left({ }^{\circ}\right.\end{array}$ & Material \\
\hline 1.00 & 39.5 & silver mica \\
\hline 1.00 & 38.3 & mylar \\
\hline 1.00 & 38 & NPO-COG \\
\hline 1.00 & 33.7 & $\begin{array}{l}\text { monolithic } \\
\text { ceramic } \\
(\mathrm{X} 7 \mathrm{R})\end{array}$ \\
\hline 1.00 & 36.9 & $\begin{array}{l}\text { ceramic } \\
\text { disc }\end{array}$ \\
\hline $0.47 \times 2$ & 31 & $\begin{array}{l}\text { monolithic } \\
\text { ceramic } \\
(\mathrm{X} 7 \mathrm{R})\end{array}$ \\
\hline
\end{tabular}

Table 1: Capacitance, $5 \mathrm{MHz}$ phase shift and material composition of measured capacitors. 


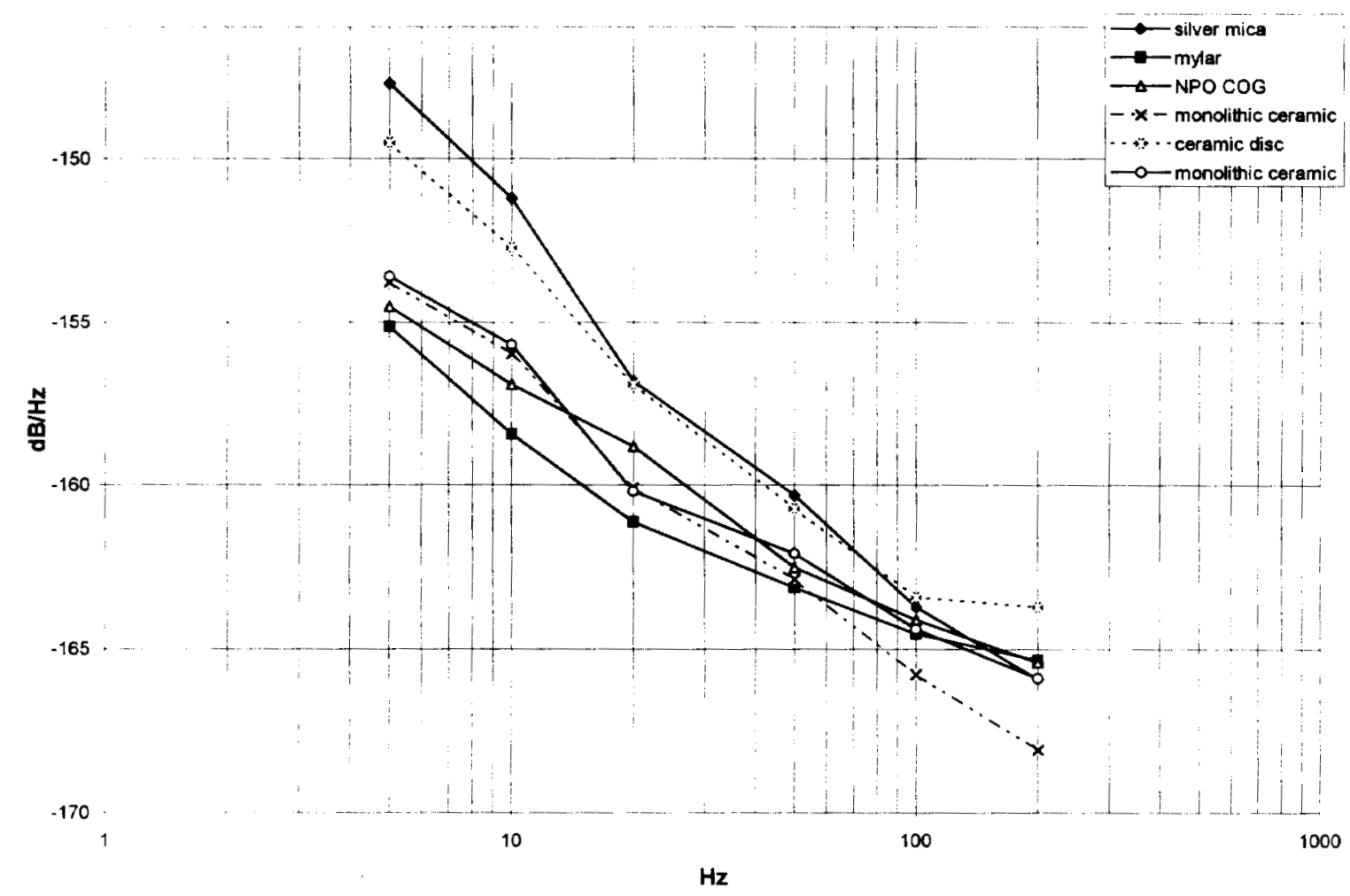

Figure 4: $\operatorname{PSD}(\triangle \mathrm{C} / \mathrm{C})^{2}$ inferred from phase shift and PM noise measurements at $5 \mathrm{MHz}$ for capacitors of different material composition.

Mylar, NPO and X7R capacitors had very low intrinsic reactance fluctuations.

\section{Inductors}

Eight different types of inductors were tested [Table 2]. All but the plastic and powdered iron core inductors are commercially available chip inductors. The plastic core inductor was hand wound over a plastic core while the powdered iron core inductor was hand wound over a commercially available powdered iron balun core. Figure 5 shows a graph of $\operatorname{PSD}(\Delta L / L)^{2}$. The air core and powdered iron had lower intrinsic fluctuations than the ferrite-core samples. The different ferrite cores exhibited large disparities in the intrinsic reactance fluctuations indicated a strong dependence on the composition of the ferrite material.

\begin{tabular}{|c|c|c|}
\hline Value (uH) & $\begin{array}{c}5 \mathrm{MHz} \\
\text { phase shift }\end{array}$ & Core Type \\
\hline 0.68 & 48.9 & ferrite 1 \\
\hline 0.39 & 52.2 & $\begin{array}{c}\text { laminated } \\
\text { ceramic }\end{array}$ \\
\hline 1.00 & 42.2 & ferrite 2 \\
\hline 0.68 & 45.3 & ferrite 3 \\
\hline 0.68 & 45.2 & air core \\
\hline 1.50 & 27.7 & $\begin{array}{c}\text { Shielded } \\
\text { with ferrite } \\
\text { core }\end{array}$ \\
\hline 0.70 & 49.3 & $\begin{array}{c}\text { plastic } \\
\text { powdered } \\
\text { iron }\end{array}$ \\
\hline 0.67 & 50.2 & . \\
\hline
\end{tabular}

Table 2: Core types, $5 \mathrm{MHz}$ phase shift and values of tested inductors. All are from different manufacturers. 


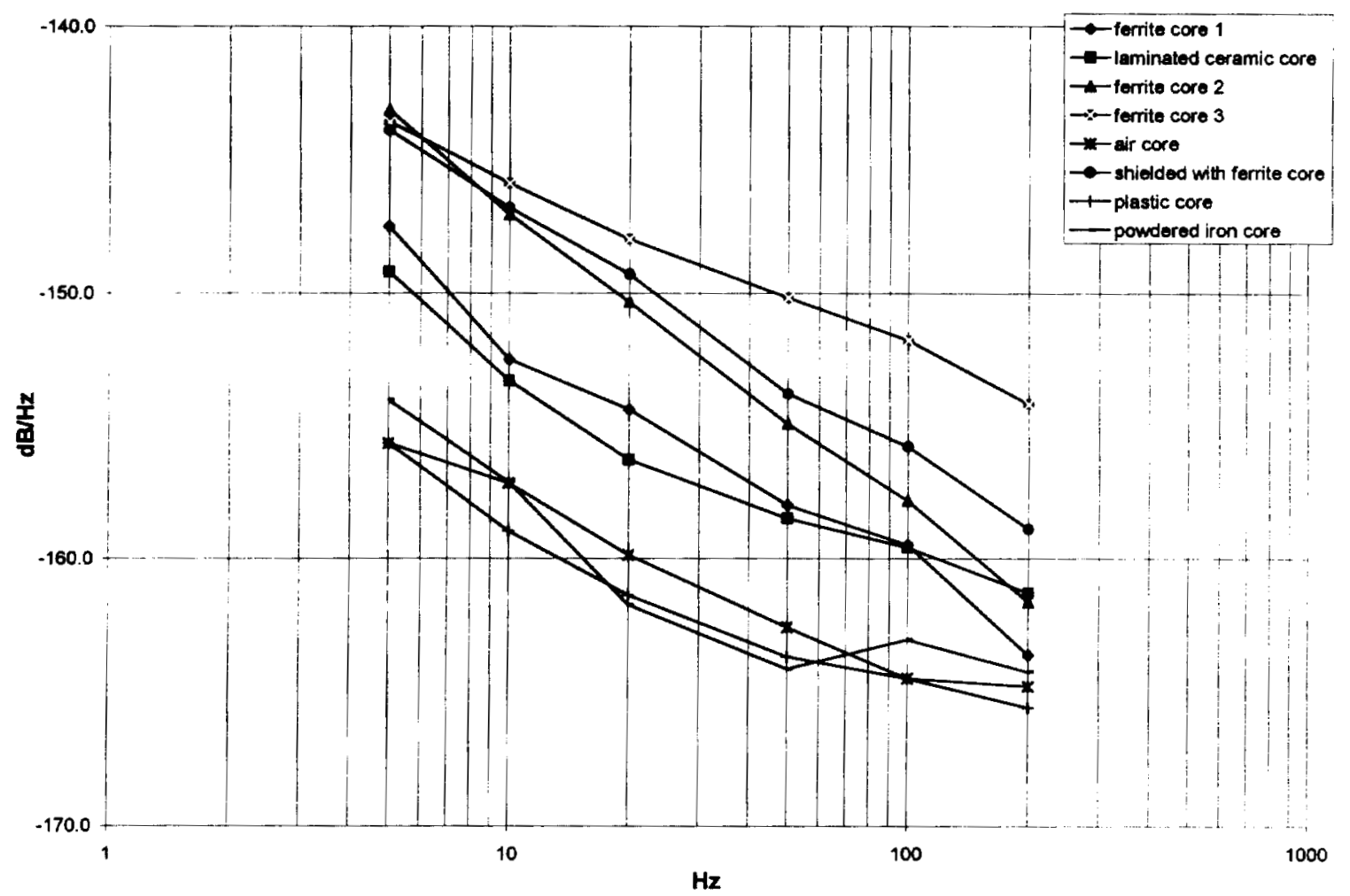

Figure 5: PSD $(\Delta \mathrm{L} / \mathrm{L})^{2}$ inferred from measured phase shift and PM noise at $5 \mathrm{MHz}$

\section{Varactors}

Leakage Current

A low bias current, low noise FET OP amp circuit with gain of $10^{9}$ was used to measure leakage current on reverse biased varactors. Although the leakage current measurements were difficult to make, repeated measurements yielded results with errors of roughly \pm 5 pA. Figure 6 shows our leakage current measurement setup.

We measured the leakage current [Fig. 7], and calculated the PSD $(\triangle \mathrm{C} / \mathrm{C})^{2}$ of two groups of diodes at different reverse bias voltages; abrupt diodes (A) [Fig. 8] , and hyper-abrupt diodes (HA) [Fig. 9]. All the diodes exhibited an increase in leakage current with reverse voltage

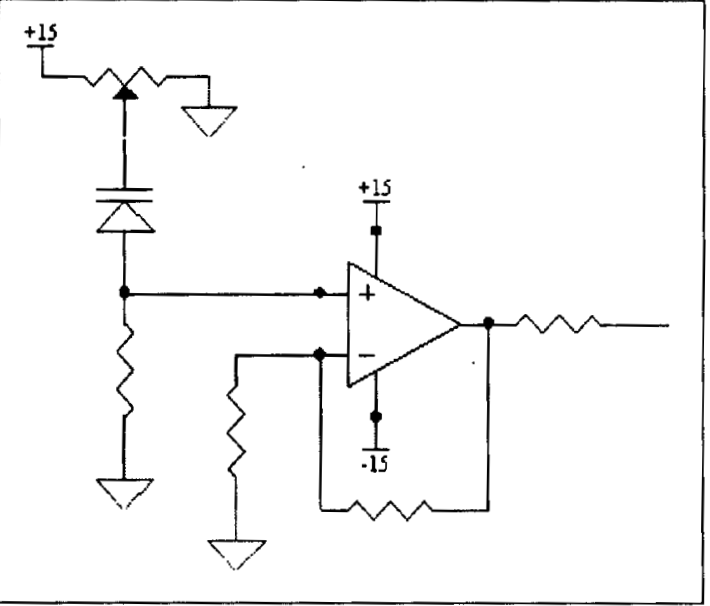

Fig. 6: Basic layout of the high gain op-amp circuit used to measure the diodes' leakage current at different reverse voltages. 


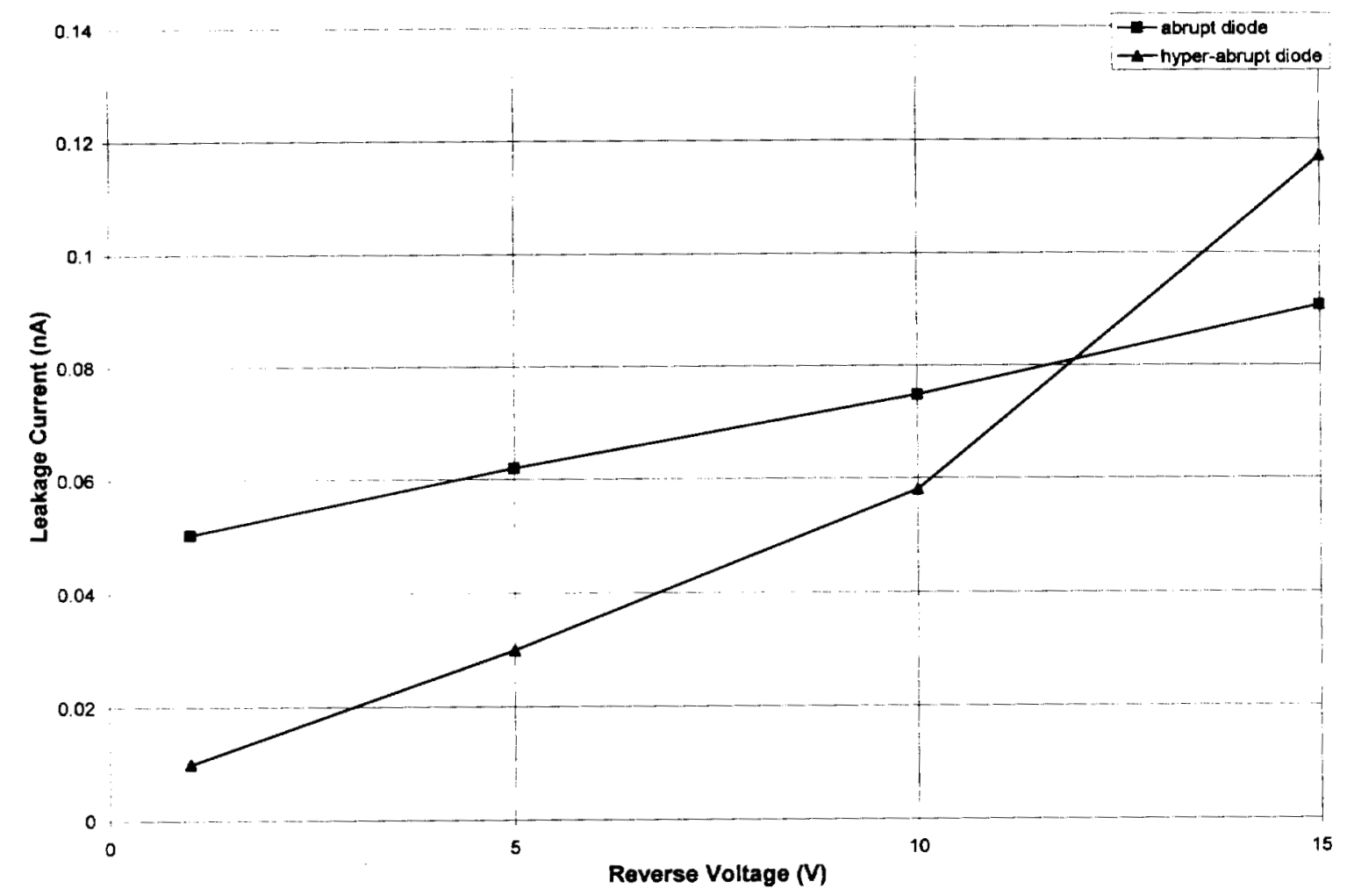

Figure 7: Leakage current vs. reverse voltage of abrupt and hyper-abrupt diodes.

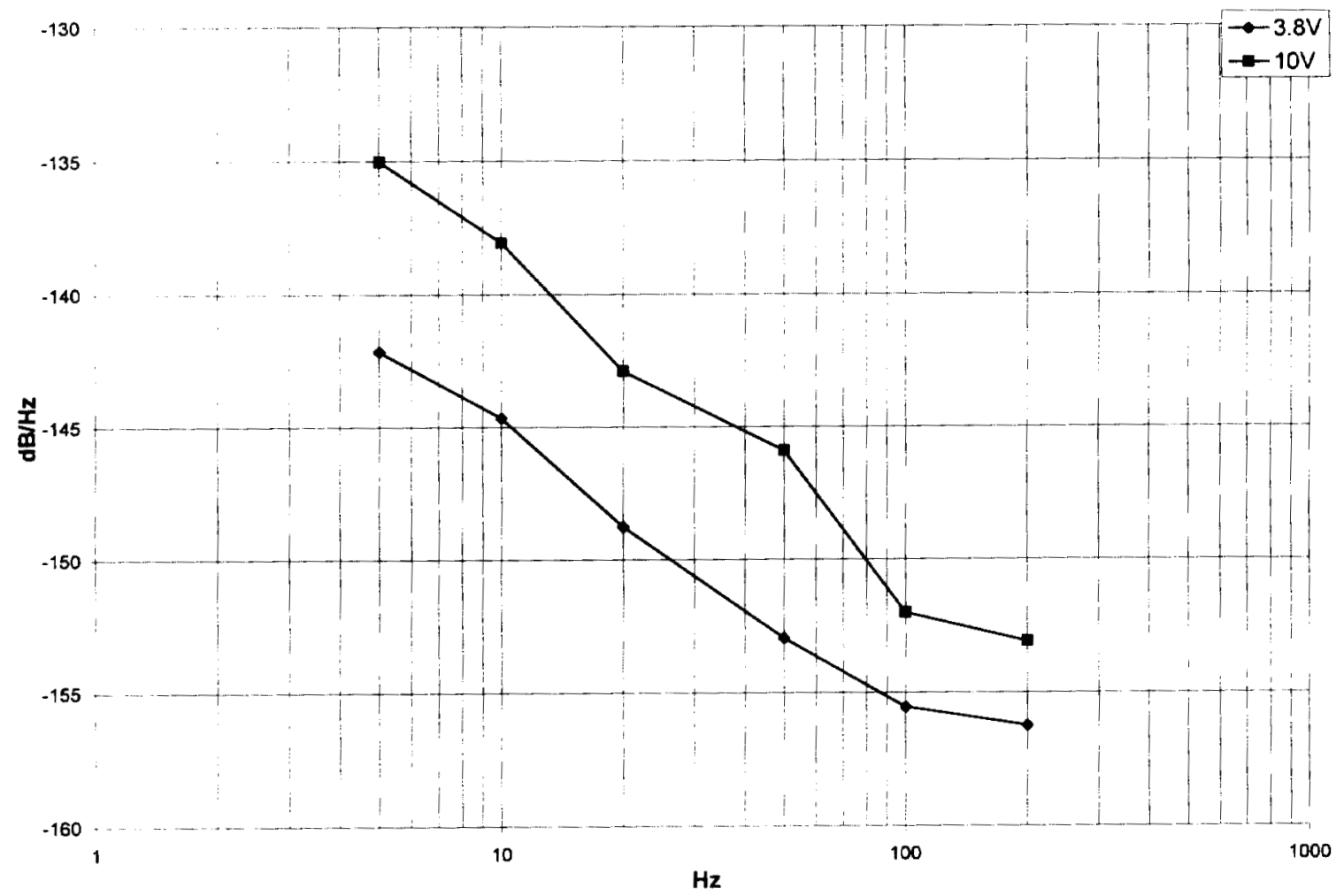

Figure 8: PSD $(\Delta \mathrm{C} / \mathrm{C})^{2}$ of Abrupt diode at 3.8 and 10 Volts. 


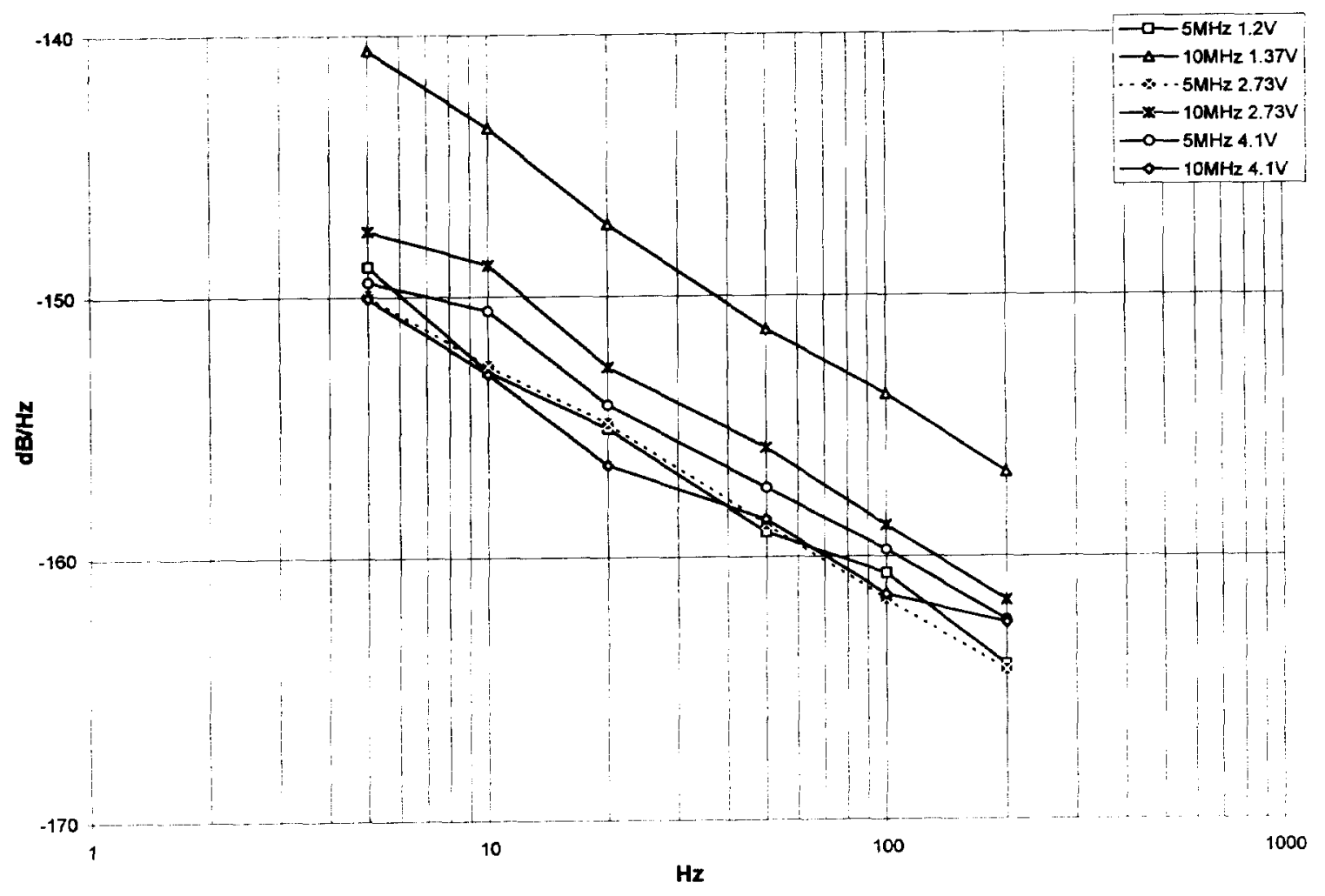

Figure 9: PSD $(\Delta C / C)^{2}$ of hyper-abrupt diode at different voltages and measurement frequencies.

The intrinsic reactance fluctuations were calculated from the phase noise and angle in the same way as the capacitor and inductor data. Ideally the intrinsic reactance fluctuations should be the same regardless of the measurement frequency. However the measurements were different for the HA diode at the same voltage, at measurement frequencies of 5 and $10 \mathrm{MHz}$. The $\mathrm{A}$ diode was measured only at $5 \mathrm{MHz}$.

The HA reactive fluctuations when measured at $5 \mathrm{MHz}$ were smaller than that of the A diode at a similar voltage [Figs. 8 and 9]. This observation agrees with our observation in Figure 8 of an increase in fractional fluctuation with reverse voltage because in both there was an increase in leakage current. The A diode has a higher leakage current at reverse voltages far from the breakdown voltage of the HA diode (15V) [Fig. 7].

\section{General Design Guidelines}

- Use monolithic ceramic or NPO capacitors when possible.

- When size is not an issue mylar capacitors are preferable

- Avoid ferrite core inductors

- When using ferrite core inductors, measure the PM noise
- Use values that produce the smallest phase shift

- Avoid pass-band resonant circuits

\section{Conclusion}

We have shown that the PM noise of a LCR circuit far from resonance depends on the angle across it as well as the intrinsic reactive fluctuations of the components. The PM noise can be at least $20 \mathrm{~dB}$ lower than the intrinsic reactive fluctuations if the angle across the component is less than $2.9^{\circ}$.

At resonance, the PM noise of a LCR circuit is $Q^{2}$ larger than the sum of the mean squared fractional fluctuations in reactance of the individual components. Therefore, using a notch filter where the notch resonance is far from the carrier frequency is preferable to using a pass-band filter at resonance.

We also deduced the intrinsic reactive fractional fluctuations of a variety of commonly available capacitors, inductors and varactors. Our capacitor, inductor and varactor data provides a basic guide for choosing individual components depending on their material. Of the tested capacitors, Mylar, NPO-COG and X7R capacitors had low intrinsic reactive fluctuations. 
Our inductor data revealed large inconsistencies in the intrinsic noise of ferrite cores from different manufacturers. However the air core and powdered iron inductors exhibited the lowest intrinsic reactive fluctuations. For low noise circuits when ferrite cannot be avoided, it is best to characterize the cores.

Our measurements on varactors indicated that intrinsic fractional reactance fluctuations are associated to leakage current. In addition, hyperabrupt diodes seemed to have smaller fractional reactance fluctuations than abrupt diodes.

Our measurements were limited to a sample size of less than five and did not take into account environmental factors such as temperature fluctuations.

\section{References}

(1) F. L. Walls, A. J. D. Clements, C. M. Felton, M. A. Lombardi, and M. D. Vanek (1988), "Extending the Range and Accuracy of Phase Noise Measurements", Proceedings of the 42nd Annual Symposium on Frequency Control, pp. 432-441.

(2) F.L. Walls (1993), "Secondary standard for $\mathrm{PM}$ and AM Noise at 5,10, and $100 \mathrm{MHz}$ ", IEEE Trans. Instrum. Meas. (Also in Proc. IEEE FCS, 1992), 42,136-143. 